\begin{tabular}{|c|l|}
\hline Title & $\begin{array}{l}\text { Numerical simulation of electron transport in electric and magnetic fields for analysis of electron temperature and } \\
\text { number density profiles measured in an argon magnetic neutral loop discharge plasma }\end{array}$ \\
\hline Author(s) & SUGA WA RA, Hirotake; OSA GA, T suy oshi; TSUBOI, Hideo; KUW A HA RA, Kiy oshi; OGA TA, Seiji \\
\hline Citation & $\begin{array}{l}\text { Japanese Journal of A pplied Physics, 49(8), 086001-1-086001-5 } \\
\text { https://doi.org/10.1143/JA P.49.086001 }\end{array}$ \\
\hline Issue Date & 2010-08-20 \\
\hline Doc URL & http://hdl.handle.net/2115/44420 \\
\hline Rights & O 2010 The Japan Society of A pplied Physics \\
\hline Type & article (author version) \\
\hline
\end{tabular}

Instructions for use 


\title{
Numerical Simulation of Electron Transport in Electric and Magnetic Fields for Analysis of Electron Temperature and Number Density Profiles Measured in Argon Magnetic Neutral Loop Discharge Plasma
}

\author{
Hirotake Sugawara*, Tsuyoshi Osaga, Hideo Tsuboi ${ }^{1}$, Kiyoshi Kuwahara, ${ }^{2}$ and \\ Seiji OGATA ${ }^{3}$
}

Graduate School of Information Science and Technology, Hokkaido University, North 14 West 9, Sapporo 060-0814, Japan

${ }^{1}$ Industrial Engineering Center, ULVAC, Inc., 2500 Hagizono, Chigasaki, Kanagawa 253-8543, Japan

${ }^{2}$ Research and Development Division, ULVAC, Inc., 2500 Hagizono, Chigasaki, Kanagawa 253-8543, Japan

${ }^{3}$ Institute of Semiconductor and Electronics Technologies, ULVAC, Inc., 1220-1 Suyama, Susono, Shizuoka

410-1231, Japan

\begin{abstract}
A Monte Carlo simulation of electron transport in electric and magnetic fields was performed to analyze experimental data of the electron temperature $T_{\mathrm{e}}$ and electron number density $n_{\mathrm{e}}$ measured in a magnetic neutral loop discharge (NLD) plasma driven in $\mathrm{Ar}$ at $0.13 \mathrm{~Pa} . T_{\mathrm{e}}$ and $n_{\mathrm{e}}$ in the vicinity of the substrate holder were measured with a triple probe, and their radial profiles had peaks at different radial positions. The simulation reproduced these peak positions well under the chosen boundary condition that the electron reflectivity of the side wall was lower than that of the reactor ceiling and the substrate holder. It was explained that the $T_{\mathrm{e}}$ peak was formed by high-energy electrons transported from the neutral loop along a separatrix of the quadrupole magnetic field and that the $n_{\mathrm{e}}$ peak consisted of electrons undergoing reciprocating motion between the reactor ceiling and the substrate.
\end{abstract}

\section{Introduction}

Magnetic neutral loop discharge (NLD) plasma is a type of inductively coupled plasma. The NLD plasma is generated along a ring called the magnetic neutral loop (NL) consisting of points of zero magnetic field. ${ }^{1-3)}$ A typical NLD plasma reactor is composed of a cylindrical vessel and three coaxial coils surrounding the vessel (see Fig. 1). The current of the middle coil flows in the opposite direction to those of the top and bottom coils to form the NL by cancelling the magnetic field there.

The NLD plasma can be operated at high electron number densities at low gas pressures, e.g., $10^{10}-10^{11} \mathrm{~cm}^{-3}$ in Ar at $0.067-0.13 \mathrm{~Pa}^{4,5)}$ This feature is desirable for finer and more economic dry etching in semiconductor fabrication. Moreover, the NLD plasma has a possibility of uniform wide-area processing under dynamic control of the radius and the vertical position of the NL by adjusting the coil currents. To develop such an advanced control scheme,

\footnotetext{
*E-mail address: sugawara@ist.hokudai.ac.jp
} 


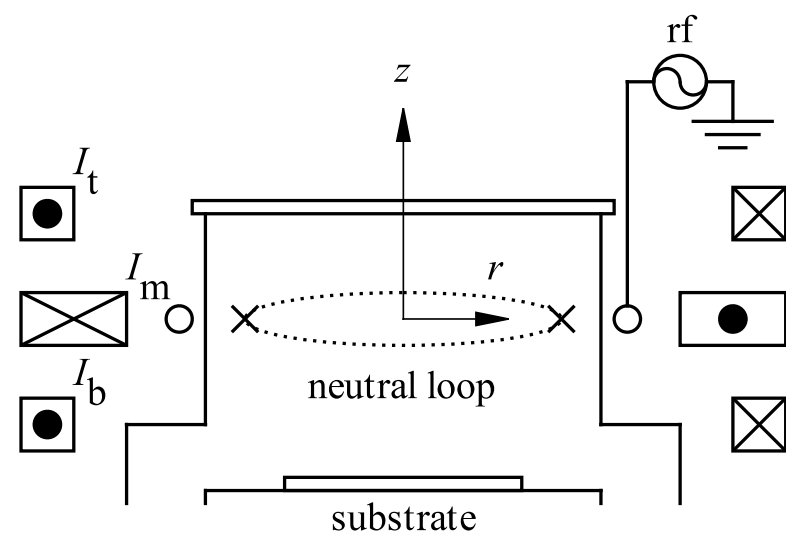

Fig. 1. Schematic of NLD plasma reactor (not to scale). The X points in the reactor represent the $\mathrm{NL}$, at which the magnetic field induced by the currents $I_{\mathrm{t}}, I_{\mathrm{m}}$, and $I_{\mathrm{b}}$ flowing in the top, middle, and bottom coils cancel each other.

it is essential to understand the structure of the NLD plasma and the transport of the species in the plasma.

In this paper, we present the results of a Monte Carlo simulation performed to analyze experimentally obtained profiles of an Ar NLD plasma. In the experiment, the electron temperature $T_{\mathrm{e}}$ and the electron number density $n_{\mathrm{e}}$ were measured in a downstream region where a substrate to be processed by the NLD plasma would usually be placed. The radial profiles of $T_{\mathrm{e}}$ and $n_{\mathrm{e}}$ were characteristic in that they had peaks at different radial positions inside of the NL. There are some reports that the peak position of $n_{\mathrm{e}}$ in the NL plane is inside the NL. ${ }^{4-6)}$ However, the inward shift of the $T_{\mathrm{e}}$ and $n_{\mathrm{e}}$ peaks in the downstream region, which may determine the etching uniformity, remains an interesting feature to be investigated. We explain the factor that induces the peak shift from the viewpoint of electron transport in the electric and magnetic fields customized for the NLD plasma.

\section{Experimental Setup and Measured Data}

The NLD plasma reactor was a quartz cylinder with an inner diameter of $43 \mathrm{~cm}$, and was equipped with three magnetic coils and a one-turn $\operatorname{rf}(13.56 \mathrm{MHz})$ antenna surrounding the cylinder coaxially. We defined the $r$ and $z$ coordinates as shown in Fig. 1, taking the origin at the center of the middle coil and the rf antenna.

The dc coil currents $I_{\mathrm{t}}, I_{\mathrm{m}}$, and $I_{\mathrm{b}}$ for the top, middle, and bottom coils were, respectively, $+18.8,-14.3$, and +18.8 A. The NL radius $r_{\mathrm{NL}}$ was about $20.5 \mathrm{~cm}$. The NL was formed near the side wall to obtain tight coupling between the NLD plasma, which can be modelled as a ring conductor, ${ }^{10)}$ and the rf antenna, as often arranged in practical operations of the NLD plasma. The magnetic field strength was about $2.0 \mathrm{mT}$ at the origin. Here, the magnetic field was set to be 1.5-2.0 times as strong as in practical operations to enhance the electron confinement by 


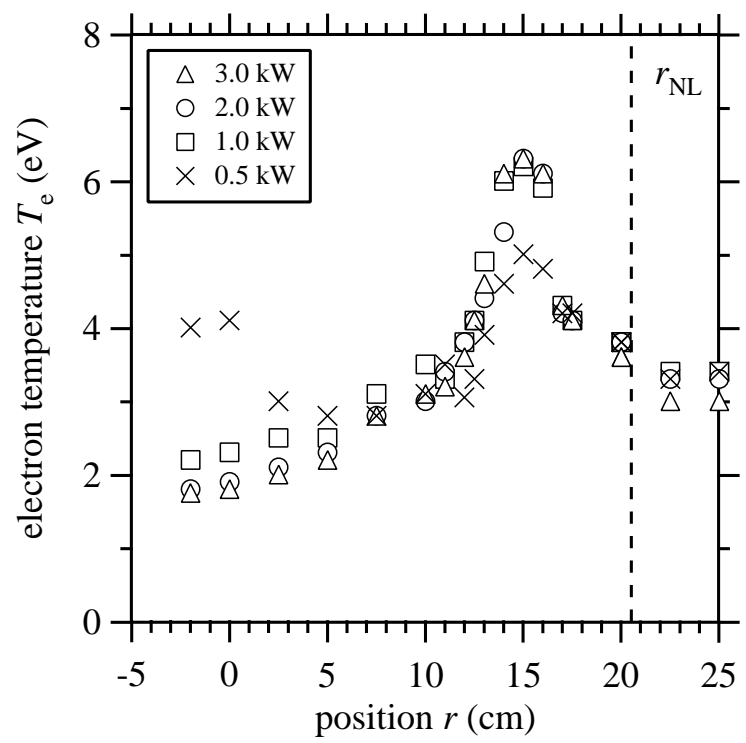

Fig. 2. Radial profile of electron temperature $T_{\mathrm{e}}$ (plotted as $k T_{\mathrm{e}}$ in $\mathrm{eV}$ with Boltzmann constant $k$ ) at $z=-15 \mathrm{~cm}$ (experiment). The broken line indicates the NL radius.

the magnetic field. $n_{\mathrm{e}}$ of the NLD plasma increases and its ring shape becomes clearcut under a strong magnetic field. The resulting high electrical conductivity of the NLD plasma enables an efficient power supply ${ }^{10)}$ which is desirable for a high throughput in material processing.

The discharge medium was $\mathrm{Ar}$ at a pressure of $0.13 \mathrm{~Pa}(1 \mathrm{mTorr}) . T_{\mathrm{e}}$ and $n_{\mathrm{e}}$ were measured using a triple probe at $z=-15 \mathrm{~cm}$ (downstream from the NL). The height of the substrate holder was adjustable, and $z=-15 \mathrm{~cm}$ was a typical substrate position.

Figures 2 and 3 respectively show the radial profiles of $T_{\mathrm{e}}$ and $n_{\mathrm{e}}$ at plasma powers $P=0.5-3.0 \mathrm{~kW}$ deposited through the rf antenna. The estimated error range for these data was about $\pm 10 \%$. Overall, the profile of $T_{\mathrm{e}}$ was independent of $P$. An exception was that $T_{\mathrm{e}}$ near $r=0$ at $P=0.5 \mathrm{~kW}$ was higher than those at higher $P$ values, but the reason behind this has not been clarified yet. On the other hand, $n_{\mathrm{e}}$ increased with $P$. It seems that the increase in $P$ enhanced not $T_{\mathrm{e}}$ but $n_{\mathrm{e}}$.

Here, let us focus on the peak positions of $T_{\mathrm{e}}$ and $n_{\mathrm{e}}$ in their radial profiles as the main discussion point of this paper. The peak positions (denoted hereafter as $r_{\text {peak,Te }}$ and $r_{\text {peak,ne }}$ ) were unchanged at approximately $r=15 \mathrm{~cm}$ and $r=12-13 \mathrm{~cm}$, respectively, in the present $P$ range. Interesting points of this result are that both $r_{\text {peak,Te }}$ and $r_{\text {peak,ne }}$ were smaller than $r_{\mathrm{NL}}=20.5 \mathrm{~cm}$ and that $r_{\text {peak }, n \mathrm{e}}<r_{\text {peak,Te }}$. Since it is considered that electrons gain energy from the electric field near the NL, where the magnetic field is weak, the peak of $T_{\mathrm{e}}$ would appear around $r=r_{\mathrm{NL}}$ if in the NL plane $(z=0)$. The peak shift occurs during the electron transport from $z=0$ to $z=-15 \mathrm{~cm}$. Its mechanism is explained in terms of the magnetic field configuration in the succeeding section. 


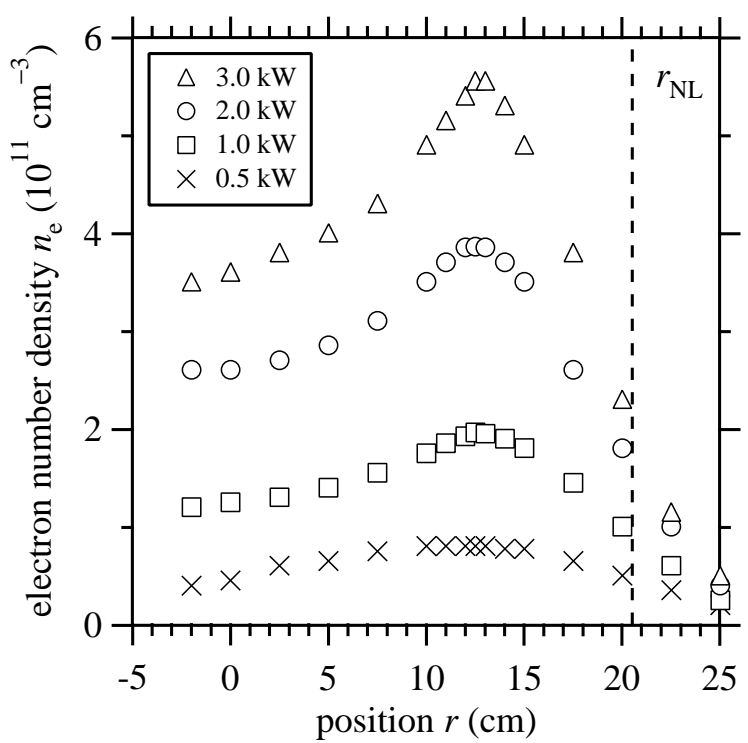

Fig. 3. Radial profile of electron number density $n_{\mathrm{e}}$ at $z=-15 \mathrm{~cm}$ (experiment). The broken line indicates the NL radius.

\section{Analysis}

\subsection{Reactor geometry and field configuration}

The dimensions of the NLD plasma reactor and the coil currents were set the same as in the experiment. The substrate holder was located at $z=-15 \mathrm{~cm}$, and its diameter was $41.0 \mathrm{~cm}$. Figure 4 shows the magnetic field in the reactor calculated from the coil currents on the basis of Biot-Savart's law and some examples of the magnetic field lines. The magnetic field around the NL (the X point) at $(r, z)=(20.5 \mathrm{~cm}, 0)$ is quadrupole. The curves connecting to the NL are called separatrices and are the boundaries between the four regions of the quadrupole magnetic field. One of the separatrices reaches the substrate holder, and its foot is at $(15.1 \mathrm{~cm},-15.0 \mathrm{~cm})$. It is noteworthy that the experimental result $r_{\text {peak }, T \mathrm{e}}=15 \mathrm{~cm}$ agrees well with this foot position. This point is mentioned again in the discussion on the simulation result of $T_{\mathrm{e}}$.

The electric field was applied in the azimuthal direction with a sinusoidal waveform of $E(t)=E_{0} \sin \omega t$. The amplitude of the rf antenna current was chosen to be $30 \mathrm{~A}$, and the value of $E_{0}$ was about $1.3 \mathrm{~V} \mathrm{~cm}^{-1}$ at the NL.

\subsection{Simulation model}

The electron motion was traced by a Monte Carlo method. The Runge-Kutta fourth-order method was adopted to calculate the electron trajectories, as in a previous work. ${ }^{11}$ ) The occurrence of the electron-Ar collisions was determined by random numbers, and the set of the electron collision cross sections of Ar referred to for the evaluation of the collision probability was taken from ref. 12. The set includes collision cross sections of momentum transfer, 


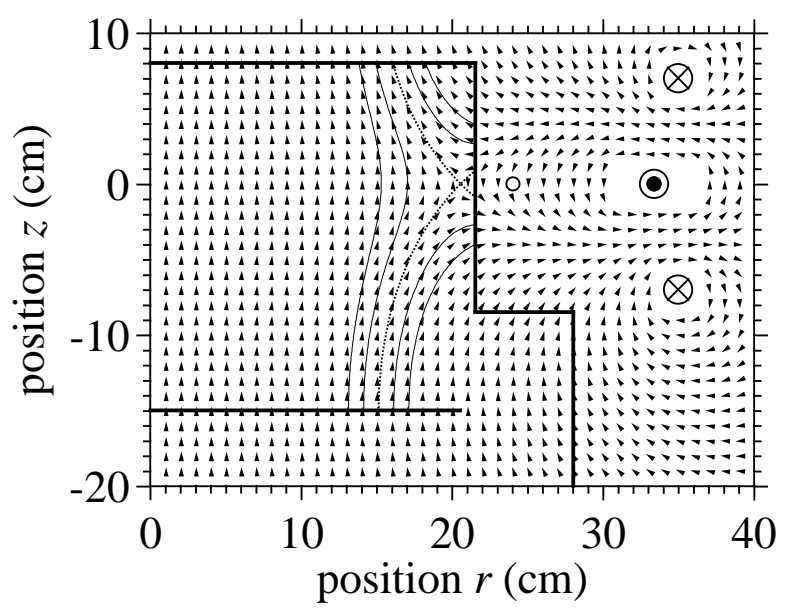

Fig. 4. Direction of magnetic field in the plasma reactor. The solid curves are representative magnetic field lines near the neutral loop. The dotted curves represent the separatrices, which are the boundaries separating the four regions of the quadrupole magnetic field around the neutral loop. The thick solid lines indicate the reactor walls and substrate holder.

excitation, and ionization. A time-saving method for judgement of the electron collisions, ${ }^{13)}$ which is suitable for the present low-pressure condition $(0.13 \mathrm{~Pa})$, was adopted to reduce the computational load.

To analyze the electron transport from the near-NL region, where the electron energy gain occurs, to the downstream region, the initial electrons were released isotropically from the near-NL region with initial energies subjected to a Maxwellian distribution of a mean energy of $1.0 \mathrm{eV}$, and they were traced for $100 \mathrm{rf}$ cycles (7 $400 \mathrm{~ns}$ ). The number of electrons sampled at the end of the simulation time was more than $10^{5}$, which includes the secondary electrons generated by ionization during the simulation.

Unlike some preceding simulations in which perfect absorption at the boundary was assumed, ${ }^{8,14)}$ we considered electron reflection at the reactor wall. This was because the number of electrons decreased rapidly and it was difficult to keep the electron population in the NLD plasma in the preliminary simulations under the conventional boundary condition. Fujimoto et al. ${ }^{15)}$ and Itoh et al. ${ }^{16)}$ reported that the reflectivities of $\mathrm{Au}, \mathrm{Al}$, and $\mathrm{Cu}$ ranged around 0.6-0.9. Golubovskii et al. ${ }^{17)}$ modelled the behaviour of charged particles on the surface of a dielectric in an analysis of barrier discharge. However, quantitative data on the electron reflectivity at the reactor wall applicable to the present simulation is rarely found in the literature. Thus, the value of the reflectivity $R$ was treated as an adjustable parameter.

In this paper, we present simulation results under $R=0.99$ for the reactor ceiling and the substrate holder and $R=0.50$ for the side wall. The electron reflection was treated to be inelastic, and the ratio $\eta$ of the energy remaining for the reflected electrons was assumed to be 0.5 . This condition, which is an example of conditions that allow the good reproduc- 
tion of the characteristic profiles of the experimental results, was chosen from among various combinations of $R$ and $\eta$ values examined in the preliminary simulations. The electron loss at the boundary was regarded to be the total of electron absorption/adsorption and recombination with positive ions. Furthermore, the electron reflection may effectively contain electron desorption, emission of secondary electrons, and even a change in the flight direction in the vicinity of the reactor wall owing to the repulsive force from the negative charge accumulated on the wall. Since the electron adsorption and desorption would occur continuously in a steady state, they would resemble inelastic reflection in total and the $\eta$ value may involve the energy loss upon adsorption. Note here that the present condition is provisional since the $R$ and $\eta$ values include quantitatively unknown factors and they may vary when the simulation model is improved. Experimental data for these factors are required to analyze the boundary condition in more detail.

\subsection{Simulation results and discussion}

Figures 5 and 6 show the distributions of the mean electron energy $\langle\epsilon\rangle$ and the electron number density $n_{\mathrm{e}}$ in the vessel. Here, $\langle\epsilon\rangle$ can be related to $T_{\mathrm{e}}$ as $\langle\epsilon\rangle=\frac{3}{2} k T_{\mathrm{e}}$ with the Boltzmann constant $k$. On the other hand, the value of the calculated $n_{\mathrm{e}}$ is arbitrary. Note in the following comparison that $n_{\mathrm{e}}$ represents the relative concentration of electrons in the vessel. Since only the electrons originating in the initial electrons released from the nearNL region were sampled, the factor for obtaining the absolute value of $n_{\mathrm{e}}$ was not included. Nonetheless, the key profiles of the experimental data that $T_{\mathrm{e}}$ had its peak at the foot of a separatrix and that the peak of $n_{\mathrm{e}}$ shifted further inward than that of $T_{\mathrm{e}}$ were successfully reproduced by the simulation.

In Fig. 5, it is observed that electrons near the NL have high mean energies. This feature agrees with the well-accepted understanding that electrons in the near-NL region can be accelerated efficiently by the electric field because the magnetic field is weak there. The magnetic field line around which an electron gyrates guides the electron, ${ }^{14)}$ and magnetic field lines passing through the near-NL region gather in a separatrix as they leave from the NL. As a result, the electrons accelerated in the near-NL region are likely to be transported to the downstream region along the separatrix. These electrons formed the peak of $\langle\epsilon\rangle$ at the foot of the separatrix. It is considered that the peak of $T_{\mathrm{e}}$ observed in the experiment was due to the high-energy electrons transported from the near-NL region along the separatrix.

The $n_{\mathrm{e}}$ distribution in Fig. 6 was formed mainly by those electrons in reciprocating motion between the reactor ceiling and the substrate holder. Here, for the convenience of explanation, let us define the inner, outer, upper, and lower regions as the four regions separated by the separatrices (see Fig. 7). Since the magnetic field lines in the upper, lower, and outer regions cross the side wall, the reflectivity of which was set to be lower than that of the reactor 


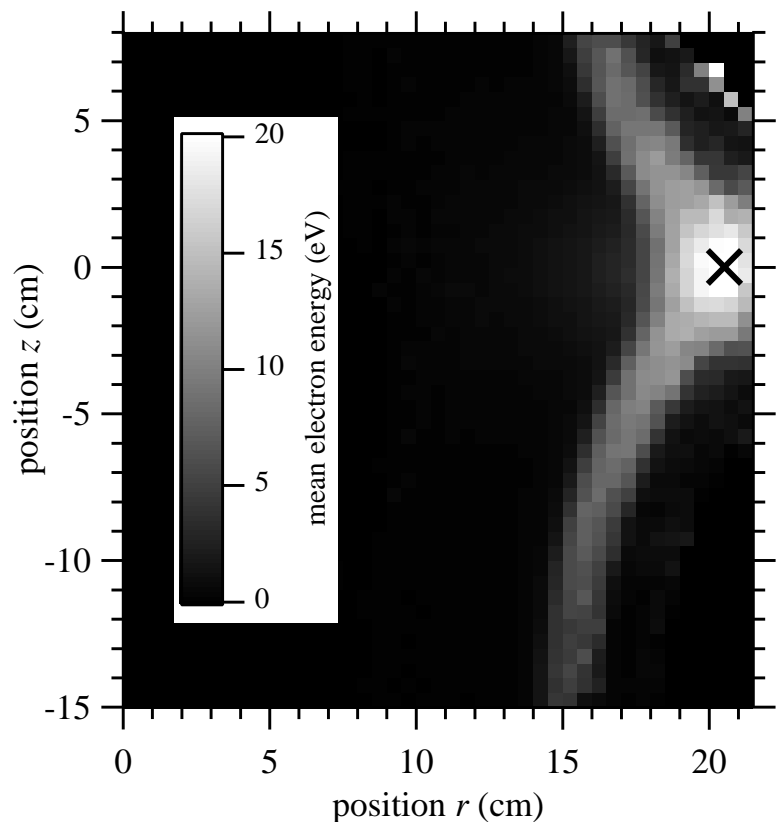

Fig. 5. Spatial distribution of mean electron energy $\langle\epsilon\rangle$ in the NLD reactor (simulation). The $\mathrm{X}$ represents the position of the neutral loop.

ceiling and substrate holder, the electrons in these three regions tended to be captured at the side wall. On the other hand, electrons moving along magnetic field lines in the inner region remained in the plasma and became the majority. Figure 8 shows examples of the electron loci in the inner and lower regions. A reasonable explanation seems to be that the inward shift of the $n_{\mathrm{e}}$ peak from the foot of the separatrix is due to the decrease in the electrons in the lower region.

Figure 9 shows the spatial distribution of the energy density $n_{\mathrm{e}}\langle\epsilon\rangle$. This quantity is considered to have a correlation with the optical emission from the NLD plasma; the emission intensity would be more or less proportional to the electron population and it would increase with the electron energy. Whereas $n_{\mathrm{e}}$ in Fig. 6 has a dip at $(r, z)=(16 \mathrm{~cm}, 0), n_{\mathrm{e}}\langle\epsilon\rangle$ has a single peak at that position. The dip of $n_{\mathrm{e}}$ was a result of the short residence time of the electrons in the near-NL region where the electrons can easily pass through without spending time on gyration. However, high values of $\langle\epsilon\rangle$ near the NL compensated the dip. The peak of $n_{\mathrm{e}}\langle\epsilon\rangle$ appeared inside of the NL. This feature agrees with a previous report ${ }^{4)}$ that the radius of the light ring core in an NLD plasma was less than the NL radius.

\subsection{Possibility of etching rate control}

The function of the separatrix to lead energetic electrons towards the substrate would play a key role in controlling the rate and uniformity of the plasma processing. An M-shaped radial profile of the sputtering/etching rate was obtained in $\mathrm{Ar}^{4)}$ and $\mathrm{C}_{3} \mathrm{~F}_{8} / \mathrm{Ar} / \mathrm{H}_{2}{ }^{2,18)} \mathrm{NLD}$ plasmas; i.e., the rate had two peaks (even within an acceptable uniformity in practical operation 


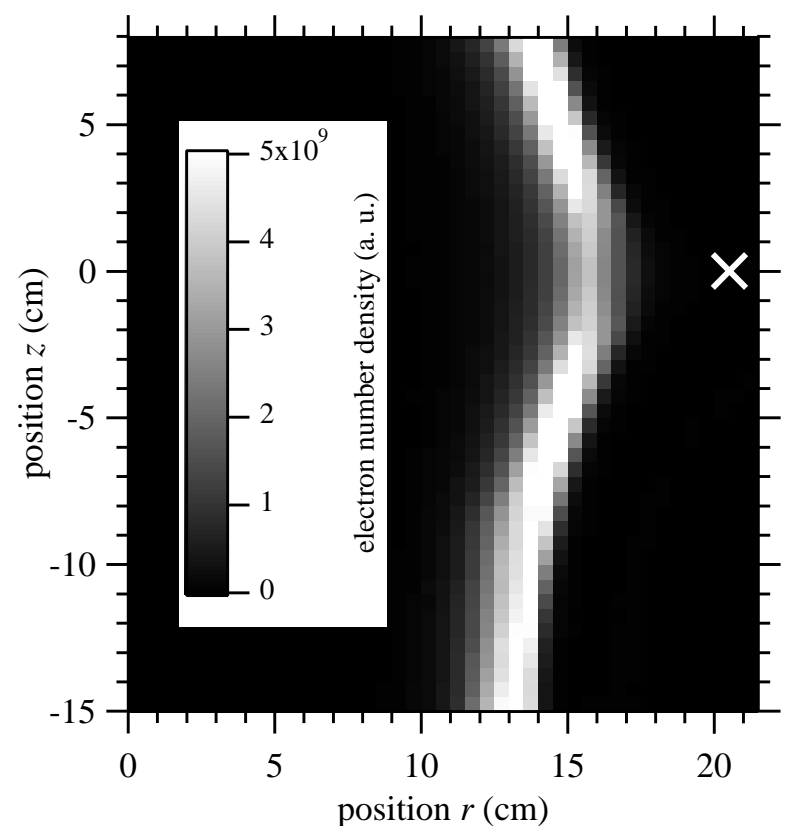

Fig. 6. Spatial distribution of electron number density $n_{\mathrm{e}}$ in the NLD reactor (simulation). The $\mathrm{X}$ represents the position of the neutral loop.

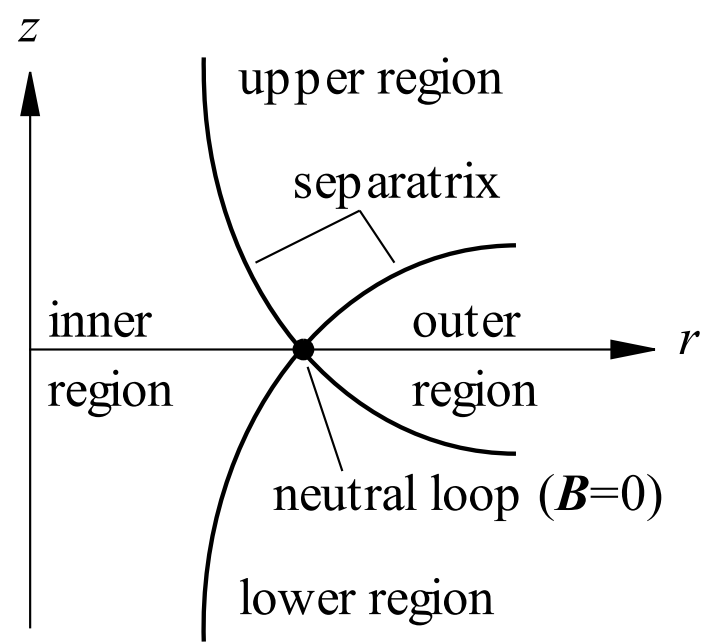

Fig. 7. Four regions defined around the neutral loop.

conditions) at symmetric positions along the diameter of an 8-inch $\mathrm{Si}$ wafer. This profile seems similar to those of $T_{\mathrm{e}}$ and $n_{\mathrm{e}}$ reported in this paper. Since higher $T_{\mathrm{e}}$ and $n_{\mathrm{e}}$ would lead to the production of more etching species because of enhanced dissociation and ionization, it is probable that the etching rate is high near the foot of the separatrix. By sweeping over a substrate with the foot of the separatrix, which can be controllable via the coil currents, uniform processing would be realized.

In conventional investigations of NLD plasmas, the NL has mainly been focused on as 




Fig. 8. Examples of typical loci of electrons released from the NL. The broken curves represent the separatrices.

the object to be controlled since the NL position governs the power deposition through the coupling between the NLD plasma and the rf antenna. We newly point out here the possibility of uniform etching by dynamic control of the separatrix and its foot position.

For a more detailed discussion, further analyses considering the production and transport of etching species would be necessary. This issue is left for future work.

\section{Conclusions}

We performed a Monte Carlo simulation of electron transport in electric and magnetic fields arranged to model an NLD plasma for material processing. The spatial distribution of the mean electron energy $\langle\epsilon\rangle$ and the electron number density $n_{\mathrm{e}}$ formed by the electrons released near the NL were compared with the experimental data of the electron temperature $T_{\mathrm{e}}$ and $n_{\mathrm{e}}$ measured at $z=-15 \mathrm{~cm}$, which is a typical position at which a substrate is placed.

The peak position of the measured $T_{\mathrm{e}}$ at $z=-15 \mathrm{~cm}$ agreed well with that of the calculated $\langle\epsilon\rangle$. This position was close to the foot of the separatrix. It was explained that the peak was formed by the high-energy electrons led from the near-NL region, where the electron energy gain occurs under a weak magnetic field, to the downstream region along the separatrix. The peak position of the measured $n_{\mathrm{e}}$, which was further inside than that of $T_{\mathrm{e}}$, was successfully reproduced by the simulation under the assumption that the electron reflectivity of the side wall was lower than that of the reactor ceiling and the substrate holder. It was suggested that the peak was formed by electrons in a reciprocating motion between the reactor ceiling 


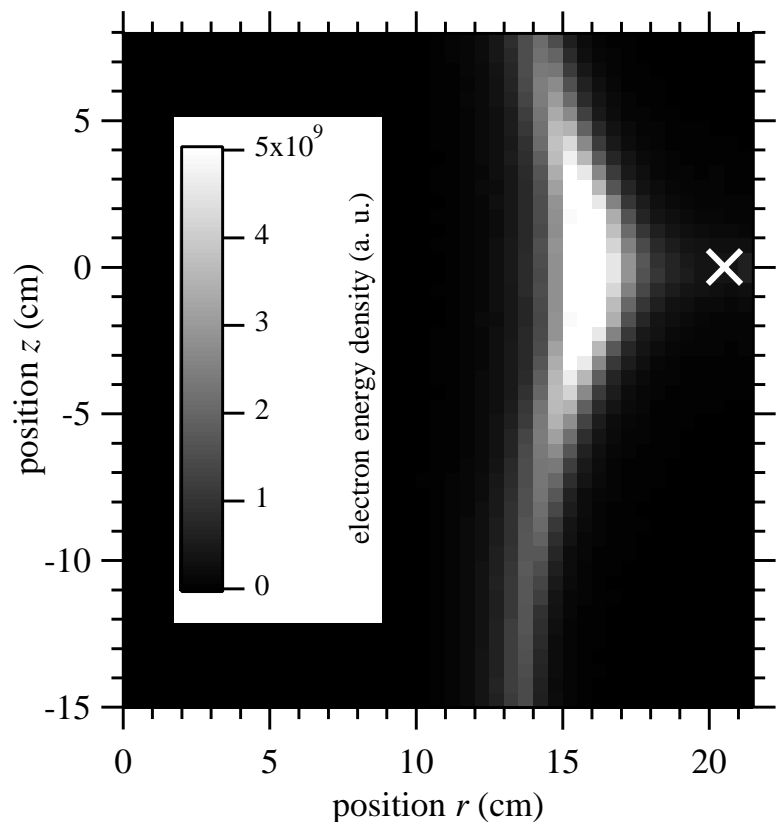

Fig. 9. Spatial distribution of energy density $n_{\mathrm{e}}\langle\epsilon\rangle$ (simulation). The $\mathrm{X}$ represents the position of the neutral loop.

and the substrate holder. On the basis of these results, it was pointed out that the separatrix could be a key control object for achieving uniform processing.

The boundary condition assumed in the present analysis is provisional, but suggests the significance of electron reflection in the sustainment of the NLD plasma. To make the simulation model more realistic in the future, we must consider more factors such as the space charge field and spread electron source in addition to the NL. Furthermore, a longer simulation time would also be necessary to enable the NLD plasma to reach a steady state. However, the present model successfully gave a qualitative explanation of the factor needed to form the peaks of $T_{\mathrm{e}}$ and $n_{\mathrm{e}}$ in the radial profile in the downstream region. It seems that the present simulation model appropriately involves the primary mechanism to govern the NLD plasma, that is, electron motion under the rf electric and quadrupole magnetic fields.

\section{Acknowledgments}

HS and TO wish to thank Professor A. Murayama and Mr. Y. Sakurai of Hokkaido University for valuable discussion and encouragement. HS also thanks Professor S. Suzuki of Chiba Institute of Technology, Professor H. Akashi of National Defence Academy of Japan and Dr. A. Oda of Nagoya Institute of Technology for information on boundary conditions at metal and dielectric surfaces. The experimental data were acquired in a project of Association of Super-Advanced Electronics Technologies (ASET) that another author (HT) was engaged in. HT acknowledges Drs. Y. Hikosaka and H. Hayashi, former researchers in ASET, for their cooperation in the experiments and also thanks Mr. M. Endo of ULVAC for his cooperation 
Published source: Japan. J. Appl. Phys. 49(8) 086001 (5 pages) (2010)

Submitted to Japanese Journal of Applied Physics

in the experiments and development of the apparatus. This work was supported in part by a Grant-in-Aid from the Japan Society for the Promotion of Science. 


\section{References}

1) T. Uchida: Jpn. J. Appl. Phys. 33 (1994) L43.

2) T. Uchida: J. Vac. Sci. Technol. A 16 (1998) 1529.

3) T. Uchida, and S. Hamaguchi: J. Phys. D: Appl. Phys. 41 (2008) 083001.

4) H. Tsuboi, M. Itoh, M. Tanabe, T. Hayashi, and T. Uchida: Jpn. J. Appl. Phys. 34 (1995) 2476.

5) H. Tsuboi, T. Hayashi, and T. Uchida: Jpn. J. Appl. Phys. 36 (1997) 6540.

6) T. Sakoda, H. Iwamiya, K. Uchino, K. Muraoka, M. Itoh, and T. Uchida: Jpn. J. Appl. Phys. 36 (1997) L67.

7) T. Sakoda, H. Iwamiya, K. Uchino, K. Muraoka, M. Itoh, and T. Uchida: Jpn. J. Appl. Phys. 36 (1997) 6981.

8) T. Sakoda, Y. Okraku-Yirenkyi, Y. M. Sung, M. Otsubo, and C. Honda: Jpn. J. Appl. Phys. 40 (2001) 6607.

9) D. O'Connell, D. L. Crintea, T. Gans, and U. Czarnetzki: Plasma Sources Sci. Technol. 16 (2007) 543.

10) H. Tsuboi and S. Ogata: Jpn. J. Appl. Phys. 46 (2007) 7475.

11) H. Sugawara and Y. Sakai: J. Phys. D 41 (2008) 135208.

12) Y. Nakamura and M. Kurachi: J. Phys. D 21 (1988) 718.

13) H. Sugawara, N. Mori, Y. Sakai, and Y. Suda: J. Comp. Phys. 223 (2007) 298.

14) Y. Okraku-Yirenkyi, Y.-M. Sung, M. Otsubo, C. Honda, and T. Sakoda: J. Vac. Sci. Technol. A 19 (2001) 2590.

15) T. Fujimoto, H. Itoh, T. Hayashi, and N. Ikuta: Denki Gakkai Ronbunshi A 115 (1995) 1072 [in Japanese].

16) H. Itoh, H. Inoue, and N. Ikuta: Denki Gakkai Ronbunshi A 110 (1990) 363 [in Japanese].

17) Yu. B. Golubovskii, V. A. Maiorov, J. Behnke, and J. F. Behnke: J. Phys. D 35 (2002) 751.

18) W. Chen, M. Itoh, T. Hayashi, and T. Uchida: J. Vac. Sci. Technol. A 16 (1998) 1594. 